\title{
An Adapted Wayfinding System for Pedestrians with Cognitive Disabilities
}

\author{
Javier Gomez, Germán Montoro, Juan Carlos Torrado, and Adalberto Plaza
}

Department of Computer Engineering, Autonomous University of Madrid, 28049 Madrid, Spain

Correspondence should be addressed to Javier Gomez; jg.escribano@uam.es

Received 9 June 2015; Revised 8 September 2015; Accepted 27 September 2015

Academic Editor: Dik Lun Lee

Copyright ( 2015 Javier Gomez et al. This is an open access article distributed under the Creative Commons Attribution License, which permits unrestricted use, distribution, and reproduction in any medium, provided the original work is properly cited.

\begin{abstract}
This paper presents a novel wayfinding system adapted to people with cognitive disabilities. It adapts to the user in terms of route calculation, instructions delivery, and interface design. To do so, the system divides the calculated route into atomic instructions and uses street-level photographs at the decision points. To evaluate this approach, we compared it with a commercial navigation application on a field trial with a sample of users $(N=18)$. From the evaluation, we concluded that our system improves users' performance in terms of the number who reached the destination and were able to identify it correctly.
\end{abstract}

\section{Introduction}

Human navigation requires mobility and orientation capabilities [1]. Both of these skills are implicit in the term wayfinding, coined by Lynch [2]. This concept includes four stages in effective navigation, namely, orientation, route decision, route monitoring, and destination recognition, in other words, determining one's own location, selecting the correct direction, checking whether the course leads to the destination or not, and recognizing the destination.

In recent years, other research work has dealt with wayfinding, such as the study by Montello and Sas [3]. These authors described effective navigation as a set of coordinated movements through an environment which are orientated to reaching an objective. Therefore, orientation is a basic skill.

In order to keep oriented, human beings rely on two complementary processes: identification of reference points and dead-reckoning [4]. The first is based on the visual identification of cues and allows us to establish an exact position from a mental map of the environment. The second is based on self-perception of pace and distance walked to estimate the position. The combination of both techniques reduces error from imprecise dead-reckoning and enables us to create mental maps.

However, people with cognitive disabilities usually present limitations in both orientation and navigation skills, due to their reduced cognitive capability. Therefore, their navigation is less effective, leading to errors and increasing the probability of getting lost. These issues directly affect their independence, limiting their autonomous movement and causing them to require supervision most of the time $[5,6]$. Generally, outdoor movements are trained by a caregiver who escorts the user over a period of time and explains the route, directions, reference points, and any other related issue. This process involves time and human resources that are not always available, preventing some users from being instructed, which limits their independence.

To address this problem, we have designed, developed, and evaluated a mobile pedestrian navigation assistant for smartphones: AssisT-OUT. Our approach adapts to the user in terms of route calculation, instructions delivery, and interface design. To achieve this, we followed a user-centered design, working in close collaboration with therapists and professionals in the field of special education, who provided their views and opinions on interface design, in particular, and took an active part in the evaluation and selecting the routes and the users.

In order to adapt the guidance provided, the system divides the calculated route into atomic instructions (continue straight, turn left, and turn right) and uses streetlevel photographs of the decision points. Additionally, it implements GPS tracking and an alarm system to notify 
users about their relative position regarding the next decision point. All these techniques promote, at the same time, the duality assistance-training. That is, an assistive tool should not only provide assistance but also train the user in the necessary capabilities to perform the activity. In this case, one of the most important capabilities is landmark recognition.

Apart from the mobile application, the system integrates an authoring tool that allows caregivers to manage their users and destination points, as well as for analyzing their performance in terms of time needed and route actually followed compared to the route originally calculated.

In order to evaluate our proposal, we tested the system with real users $(N=18)$ in a real environment and compared it against a commercial navigation application (i.e., Google Maps). From the evaluation we concluded that our system produced significantly better results than the commercial application regarding the number of users who reached the destination, but they needed significantly more time to walk the proposed routes.

This paper is organized as follows: after this short introduction we present the background for the research, emphasizing assistive technologies for people with cognitive disabilities. After that, AssisT-OUT is presented. Following a detailed explanation of the approach, the evaluation process is described. Finally, we close with a discussion on the data collected and conclude with the most important ideas and future work lines.

\section{Related Work}

Current mobile platforms and application stores offer a great variety of navigation assistants. In particular, thanks to the location possibilities of available smartphones (GPS, GLONASS, or mobile/Wi-Fi signal triangulation), these applications make our trips easier, faster, or safer by giving turn-by-turn navigation instructions. In general, they allow possibilities for adaptation: route calculation depending on users' preferences (shortest route, fastest route, etc.) and some interface customization, such as color palettes for day and night or types of points-of-interest displayed. However, neither routes nor interfaces are usually adapted to users' cognitive capabilities. In other words, the way directions are delivered and presented is not adapted to meet users' needs.

Previous research works on the topic have provided different approaches and solutions, such as the "Assistive Navigation System," presented by Hervás et al. in [7]. In this work, the authors addressed route adaptation by including places that are well-known (to the user) to calculate the best route. This way, instead of delivering the shortest or fastest path, it employs known and near places to get to the destination. That is, it uses known places as reference points in order to reduce the cognitive load of the route. Nevertheless, although most of the participants preferred this new route calculation mechanism, known reference points are not always available for every route and its preparation for each user can be difficult to process.

In this same sense, "Our way" [8] proposed a novel route calculation engine based on social collaboration. By users' annotations, the system was able to adapt the route to meet users' needs in terms of accessibility. This approach is particularly interesting for users with motor disabilities (temporal or permanent), although the authors did not report any quantitative data.

Related to instructions delivery, Rispoli et al. remarked the positive value of splitting the whole task into simpler subtasks to make them easier to understand [9].

Regarding directions and route presentation, works such as [10-13] explored different mechanisms for giving directions to people with cognitive impairments, namely, using arrows, audio prompts, maps, and landmarks. In general, all authors agreed that landmarks are the best way to orient and guide these users. However, the combination of different mechanisms (i.e., landmarks and audio cues) would be even better since the message is given in different modalities and users may recognize the information more easily. Kaminoyama et al. [14] presented the "Walk Navigation System," a navigation system developed for PDAs and particularly designed to help people with dementia. It shows streetlevel photographs at the particular points of the route (e.g., intersections, places where the user has to take an action) combined with animations and arrows to make the decision easier. From their evaluation it can be concluded that the system helped users to reach the destination and they found photographs very useful. Moreover, on average, users needed less time to reach the destination compared to the time needed when using maps, but they spent more time looking at the device than at the map. However, users reported that photos should be updated since they were different from the actual view. These may be the key issues for making improvements in new developments.

Additionally, García de Marina et al. [15] explained some other problems, such as the difficulty in distinguishing between left and right (when using textual instructions) and the confusion produced by the differences between the actual view and the picture shown, that is, items that appear or do not appear.

Finally, due to the inherent danger of the activity (i.e., getting lost), Fickas et al. [16] studied the ability to recover from errors and found that people with cognitive disabilities had serious problems describing their position in order to get reoriented, so systems should include mechanisms to provide quick and straightforward assistance and location.

\section{AssisT-OUT}

Current state-of-the-art developments offer a wide range of material with which to work on improving currently available solutions. With this aim in mind, we present AssisT-OUT, a mobile system specifically designed to help people with cognitive disabilities to walk through urban environments such as towns. Route calculation, instruction delivery, and user interface are adapted to meet users' needs.

AssisT-OUT makes use of users' smartphones with two objectives: first, to locate and track users during their movements and, second, as a presentation tool to show the appropriate directions. The guidance process, according to Fallah et al. [17], can be divided into four steps: environment 


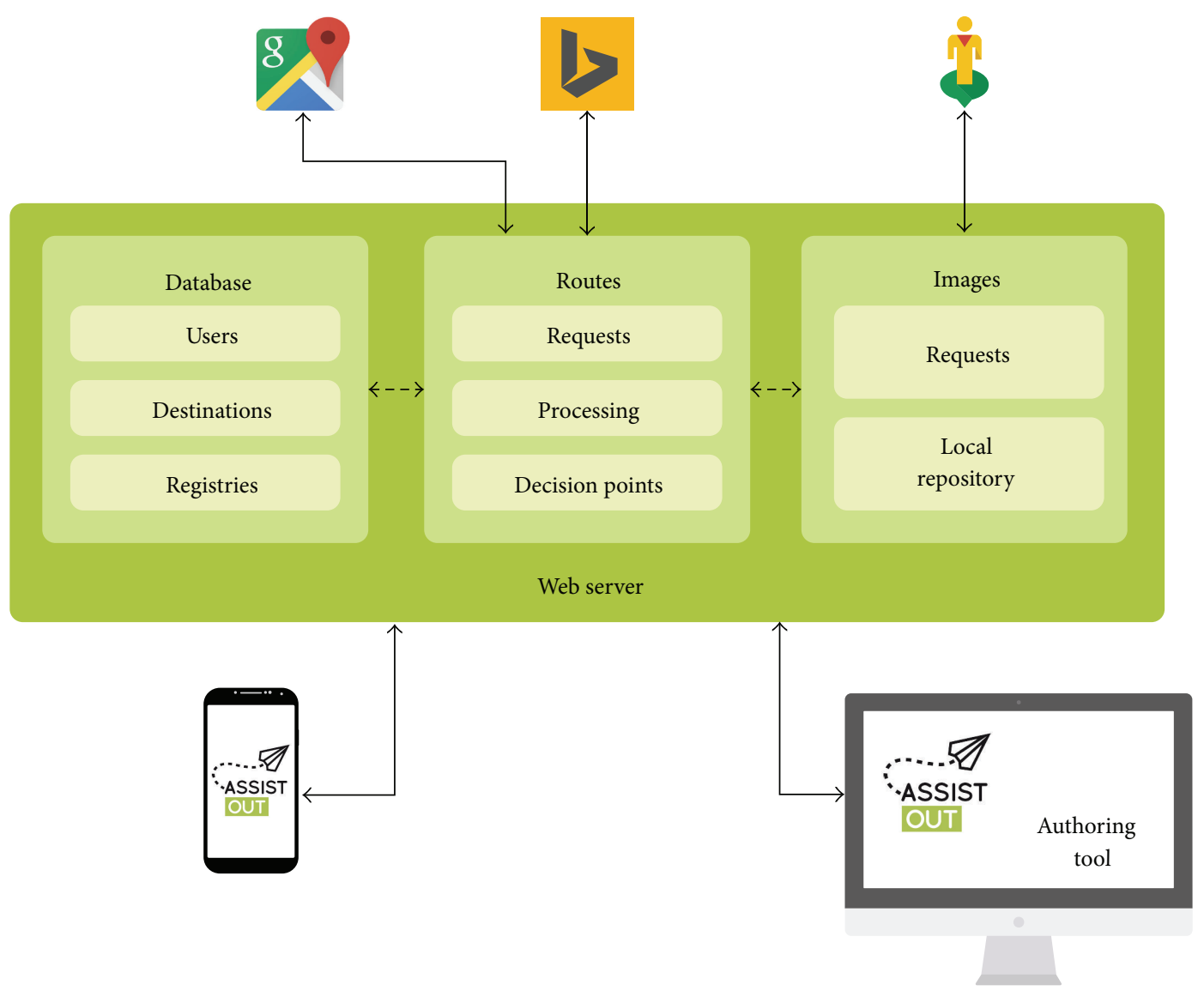

FIgURE 1: System architecture.

representation, user location, path planning, and user interaction.

3.1. Architecture. The architecture of the system is based on the client-server scheme. In Figure 1, a diagram of the system is provided. On the one hand, the mobile client locates and interacts with the user. On the other hand, the server is divided into three modules. The database stores the information related to users, their destinations and registries. The routes module communicates with third-party services (i.e., Google Maps), processes the routes, and draws up a list of directions and decision points. Finally, the images module is in charge of requesting images from Google Street View and providing the ones from the local repository (only for destination points).

The communication between the client and the server is bidirectional, since the mobile application has to provide information (user's location and registry) to the server in order to calculate the route and it also has to download information from it (e.g., destination list and routes). Additionally, the authoring tool also communicates with the server. It allows caregivers to edit users' information as well as their destinations.

3.2. Environment Representation. Since AssisT-OUT relies on available cartography services, caregivers do not have to make any environment representation beyond the destination points of their users. To establish these, they make use of the authoring tool to add, edit, or remove these points. Each point is represented by its coordinates (longitude, latitude), a name, an icon, and the street-level view of the place. The coordinates are extracted from Google Maps. Caregivers only have to look for the place on a map and add a pin at the proper location. After that, they provide the name, the icon, and the street-level photo of the destination. This last attribute can be obtained from Google Street View service or from a picture uploaded by the caregiver, providing adaptation for each specific user, if needed. Figure 2(a) shows the interface employed. When the caregiver selects a user to edit (on the left side bar), the map including that user's particular destinations is loaded and appears on the right side. The destinations are marked with a blue circle. By clicking on them, it is possible to see or edit their names or pictures.

Additionally, the authoring tool allows caregivers to supervise users' performance. Once a user finishes a route, the registry (location and time information) is processed and represented on a map. This way, a caregiver can analyze a user's performance. Figure 2(b) shows this supervision screen. For the selected user and route, it presents a map with both paths: the proposed one (solid green line) and the path followed (blue dotted line). 


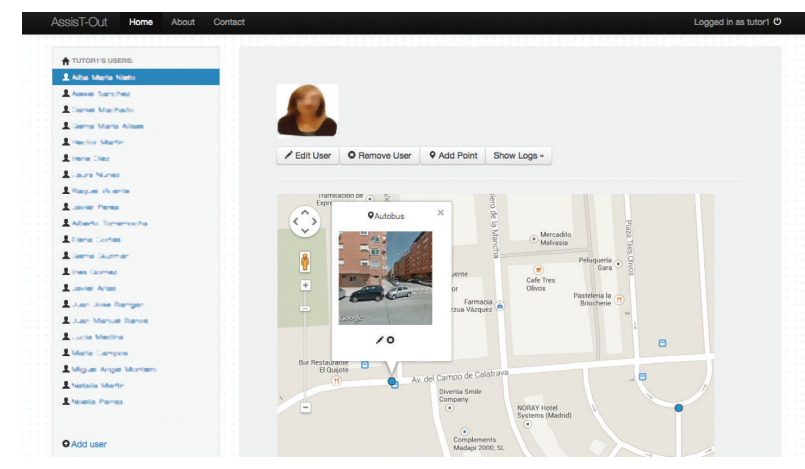

(a)

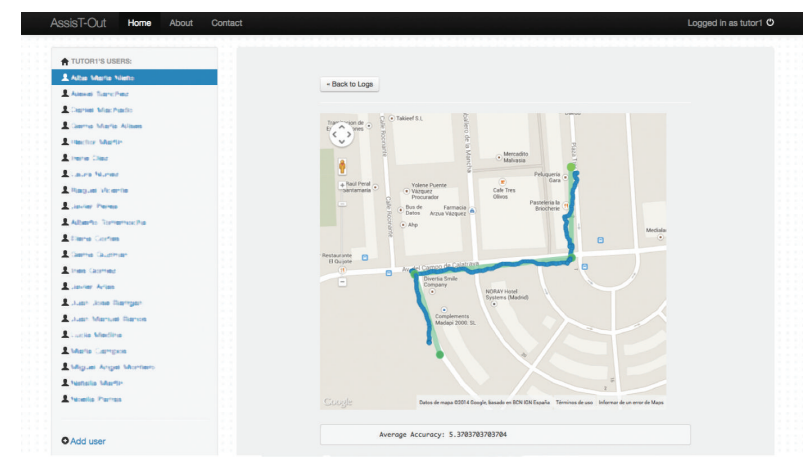

(b)

Figure 2: Authoring tool screenshots: (a) destination points edit screen. Blue circles represent the destination of the selected user. (b) Route supervision screen. Green circles correspond to the start, finish, and decision points. The solid green line represents the proposed route and the blue dotted line represents the followed route (users' names and photographs have been blurred in order to preserve their privacy).

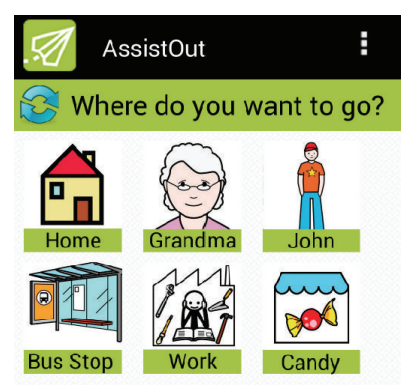

$\triangleleft \quad 0 \quad \square$

FIgURE 3: Destination selection screenshot.

3.3. Location of Users. In this case, thanks to the use of smartphones, their GPS sensors, and digital compasses, locating and tracking users are straightforward tasks.

Once users are located, the destination has to be known. Several studies have reported that people with cognitive disabilities seldom go to new places on their own [5]. Therefore, the number of possible destinations is restricted to certain places (education center, relatives' and friends' houses, work place, etc.). This list is drawn up by the caregiver and customized by means of the provided authoring tool. Each destination is represented on the smartphone screen by its icon and name. This way, the system presents the user with a list of the possible destinations to choose one. A screenshot of the interface is shown in Figure 3. Thanks to the destination icons, users can identify them easily, reducing the cognitive load of the process [18].

3.4. Path Planning. To calculate the best route, the environment must be modeled and processed. In general, navigation systems make use of graphs to model spaces and apply mathematical algorithms to calculate the shortest path between the starting and destination points. Due to the complexity and extensive work required to model a city environment, AssisTOUT makes use of third-party services such as Google Maps and Bing Maps and their APIs.

Although these services offer a certain degree of adaptation in terms of means of transportation or road preferences, the instructions provided usually include additional information, such as street names, cardinal points, and distances. This information is useful for locating decision points but they make instructions more complex, requiring a higher cognitive load to be processed to understand them. Moreover, in some situations the instructions include more than one direction, for example, "turn left and after $100 \mathrm{~m}$ your destination will be on your right." This typical cartography services instruction is too complex for people with cognitive disabilities. The adapted version of the same instruction should be divided into three simpler directions: "turn left," "go straight (for $100 \mathrm{~m}$ )," and "your destination is on your right."

This issue makes it necessary to analyze and process the chosen route. As mentioned in the previous section, dividing tasks into simpler steps would result in better understanding of the instructions by the user and, therefore, better performance. Hence, the route is divided into atomic instructions (i.e., continue straight, turn left, and turn right) so that it results in a sequence of decision points (places where users have to make a decision) and their associated instructions. In this way, the navigation process becomes a systematic task that is easy to understand and process by people with cognitive disabilities.

To facilitate the identification of decision points, every instruction is associated with a street-level photograph. This helps users to visually identify the location of these points, as well as their direction, thus considerably reducing the cognitive load required in comparison to the original instruction. To achieve this, AssisT-OUT estimates the best location for the views and requests them from the Google Street View Service. Although Google Street View can be a 


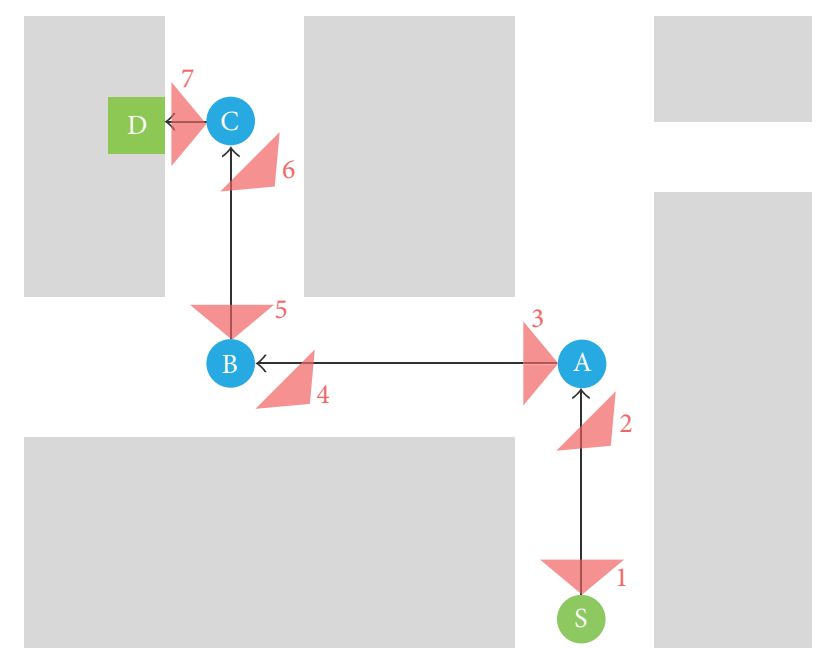

FIgURE 4: Route representation diagram, from $S$ (start) to D (destination). Circles labeled from $\mathrm{A}$ to $\mathrm{C}$ indicate decision point locations. Triangles numbered from 1 to 7 represent displayed views.

limitation due to outdated or mismatched images, it presents a major advantage: caregivers do not have to take and prepare the photographs by themselves. In other words, the image collection task can be automatized. Therefore, human and time resources required to provide the assistance are also reduced.

Figure 4 presents an example route to go from point $\mathrm{S}$ (start) to point $\mathrm{D}$ (destination), the calculated decision points are represented by circles labeled A, B, and C, and the associated views are represented by triangles, numbered from 1 to 7 . The set of instructions and views associated to the route are as follows:

(1) Turn until you see the view (point $S$, view 1).

(2) Go straight until you see the view (from point $S$ to point $\mathrm{A}$, view 2).

(3) Turn left until you see the view (point A, view 3).

(4) Go straight until you see the view (from point A to point $\mathrm{B}$, view 4).

(5) Turn right until you see the view (point B, view 5).

(6) Go straight until you see the view (from point B to point $\mathrm{C}$, view 6).

(7) You have finished (point C, view 7).

As can be seen, the views chosen to identify the decision points are located a few meters before the point and slightly oriented toward the direction the user will have to turn at the decision point. This way, all the possible references will be shown: the street, the building at the corner, and so forth, making turns and point identification simpler.

3.5. User Interaction. The breakdown into simple instructions makes navigation a systematic task, since the users have to proceed the same way all the time: read (or listen to) the instruction and execute it (walk or turn) until they find the same view that is shown on the screen.
Navigation is supported by an adapted user interface, which follows the ideas expressed in the previous section. The design process was centered on the user and supported by experts in special education. It was based on a previous design and refined according to the conclusions extracted from its evaluation [19].

It shows a photograph of the next decision point and an atomic instruction. In order to facilitate navigation, the interface also shows a progress bar and a help button. The progress bar helps to identify how far the user is from the shown decision point in a way that is graphical and easy to understand. The help button triggers an alarm, notifying the caregiver that the user feels lost. Besides, AssisT-OUT is also provided with a mechanism to warn the user as she gets close to the decision point. The mobile beeps and vibrates when this happens, relieving the user of the need to constantly pay attention to the interface while walking to the decision point.

Figure 5 shows the developed interface. At the top of the application screen, we find the instruction, for example, "go straight until you see this" (a). Under the instruction, we see the red alarm button. Below we see the picture of the next decision point and under it the progress bar. This is gray and fills in with green as the user gets close to the shown decision point. To do this, the system first calculates the distance between the decision points. After that, thanks to the GPS location, it calculates the remaining distance to the next decision point and fills the bar proportionally to the walked distance. Additionally, this progress bar is also used in turns, becoming filled when the user faces the right direction (b). This helps to alleviate the left/right confusion that many users present. In this case the sensor employed is the compass. Thanks to the information provided by Google Maps, AssisT-OUT estimates the angle that the user has to turn. Then, according to the compass measure, the bar fills green.

Finally, the interface includes two navigation buttons at the bottom of the screen. They allow users to move forward to the next step or backward to see a previous one again. Using these buttons, once a user reaches a decision point (c), she can tap on the forward button to get the next instruction with the next decision point. In order to prevent the user from skipping steps, a location awareness mechanism has been added to the forward button. This keeps it disabled until the user gets close enough to the decision point (a).

In addition to the graphical interface, AssisT-OUT provides automatic text-to-speech conversion of instructions, so users who cannot read are still able to use it.

\section{Evaluation}

The evaluation was carried out with two main objectives: on the one hand, to test the correct operation of AssisT-OUT in a real environment on users with cognitive disabilities and, on the other hand, to compare it with standard commercial navigation software: Google Maps application, available for Android smartphones at Google Play.

Therefore, we conducted an evaluation with 18 users with cognitive disabilities. All participants were students of 


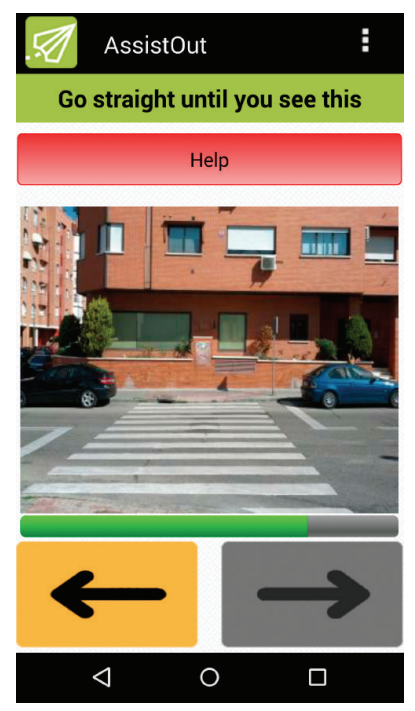

(a)

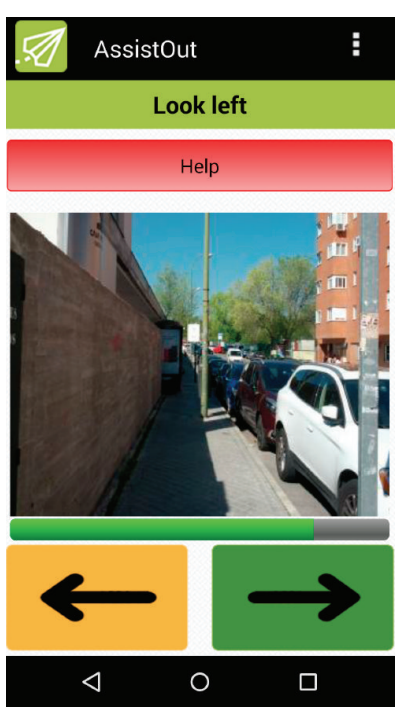

(b)

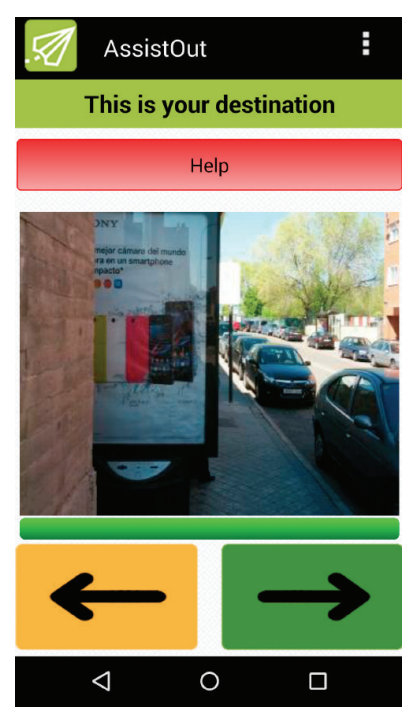

(c)

FIGURE 5: Screenshots of the navigation interface. (a) Before arriving at a decision point. (b) Turning left at the decision point. (c) Arriving at the destination.

courses at the Madrid Down Syndrome Foundation. In these courses, students are trained in job-related skills (generally, office assistant), but also social and behavioral skills and, of course, skills required for moving through different environments.

On average, users were 23.72 years old (stdev $=3.66$ ). Eleven of them were male and seven were female. Since we wanted to test AssisT-OUT as a universal tool, the recruited participants presented different cognitive levels and capabilities. In particular, one of the participants had limited vision. Regarding their relation to technology, all participants had a mobile phone, although only 11 out of the 18 reported that it was a smartphone. Finally, 7 of them used navigational applications or public transport applications (e.g., for knowing the time remaining when waiting for a bus). Therefore, there was a certain variability in the technological profiles as well.

4.1. Methodology. The evaluation process involved different techniques such as video recordings and analysis, questionnaires, focus groups, or thinking aloud. In this way, we were able to collect both qualitative and quantitative data to evaluate AssisT-OUT and compare it to Google Maps.

In order to perform the evaluation efficiently, a counterordered repeated measures experiment design was proposed. This incomplete factorial design allows comparing tools, methods, or interfaces with a minimum number of sessions but keeping user, task, and tool polarizations away. In this case study, we divided the 18 participants into two heterogeneous but similar groups. That is, both groups were composed of users with different skills and levels but, as a group, they were equivalent. We also defined two different routes and assigned them to both groups and tools. Table 1 summarizes the distribution of groups, routes, and applications.
TABLE 1: Distribution of groups, routes, and applications during the evaluation sessions.

\begin{tabular}{llcc}
\hline & & Group A & Group B \\
\hline Session 1 & Route 1 & Google Maps & AssisT-OUT \\
Session 2 & Route 2 & AssisT-OUT & Google Maps \\
\hline
\end{tabular}

Both routes started from the education center and finished at a news-stand and a candy store, respectively. The distance on both routes was $300 \mathrm{~m}$ approximately, making them similar on this account. Figure 6 presents a diagram of both routes: (a) corresponds to route 1 and (b) to route 2. As can be seen, both routes have the same number of intersections (4). However, route 1 includes three turns (left, right, and left) while route 2 includes only two (right, left).

In order to compare both routes in detail, the Richter and Duckham route complexity analysis was carried out [20]. This metric evaluates routes' simplicity by scoring their crossings and turns. Table 2 summarizes the possible values.

From the analysis of their turns and crossings, we obtained the scores summarized in Table 3. In light of the results, although both routes' scores were similar, route 1 scored slightly higher.

However, the analysis did not consider the open area on route 2, a small paved park with trees that delimited pedestrian streets. A schematic view of the area is shown in Figure 7. The solid green arrow indicates the route that should be followed, while the dashed line arrow indicates other possible paths. Therefore, this should be included in the analysis, since it added difficulty to the route. Considering this, 3 extra slots should be added to the score for route 2, leading to very close values for both routes. On these grounds, we assumed them to be equivalent in terms of difficulty. 


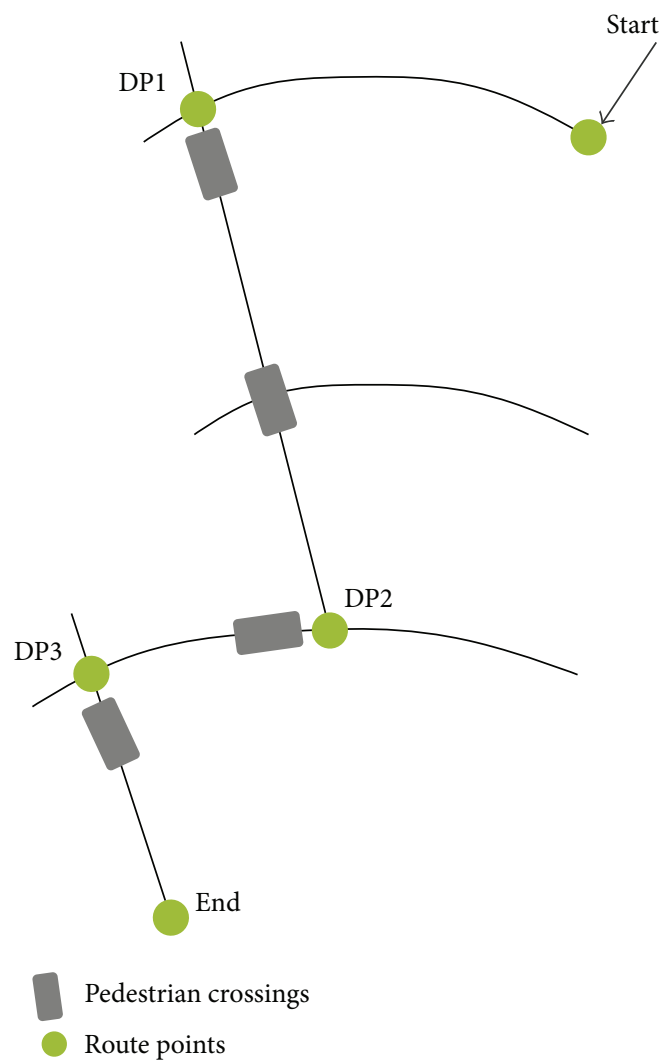

(a)

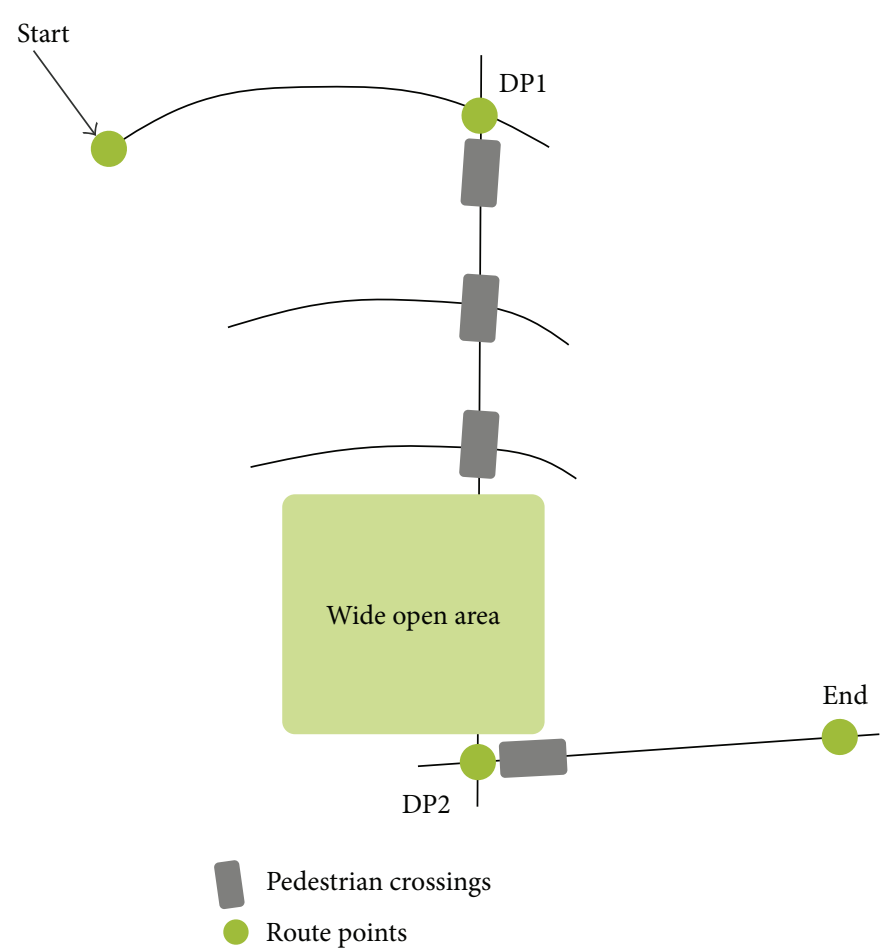

(b)

FIGURE 6: Diagrams of the route followed in the evaluation, corresponding (a) to route 1 and (b) to route 2.

TABLE 2: Scores of the different intersections, adapted from [20].

Direction
Turn at a
T-intersection
intersection)
Turn at a complex
intersection

The evaluation took place during class time. Each participant was asked to leave the class individually and go to the main door of the building. Before walking the route, they received a short training session on the application, consisting in a short walk to a nearby bus stop. During this training they were assisted by the smartphone and a researcher. The researcher showed them all the features of the application and gave them a short explanation on the guidance mode of the corresponding application (Google Maps or AssisT-OUT). Once they got to the bus stop and all their doubts were solved, they started the test route.

During the walk, users were escorted by two researchers, who walked a few meters behind them. One was tasked to help the user in case of a mistake or any other situation while the other recorded the session to obtain a third-person view of the user during the test. Additionally, users were asked to wear a head-mounted camera to record a first-person view. These videos, in combination with the AssisT-OUT registries, were analyzed afterwards.

To start the walk, users received the smartphone with the application installed. Besides, in the case of Google Maps, the destination was preset by a researcher, as otherwise some of the participants would not have been able to choose the destination and carry out the evaluation. This marked a difference with regard to AssisT-OUT. The initial state of the Google Maps application is shown in Figure 8. As can be seen, the destination was preselected before giving the phone to the user. 
TABLE 3: Complexity analysis of both routes.

\begin{tabular}{lccc}
\hline \multicolumn{1}{c}{ Route 1 } & & & Route 2 \\
\hline Turn left $($ complex $) \operatorname{deg}(v)=4$ & 9 & Turn right $(\operatorname{complex}) \operatorname{deg}(v)=4$ & 9 \\
Straight on & 1 & Straight on & 1 \\
Turn right $(\mathrm{T})$ & 6 & Straight on & 1 \\
Turn left $($ complex) $\operatorname{deg}(v)=4$ & 9 & Turn left $(\operatorname{complex}) \operatorname{deg}(v)=4$ & 9 \\
Total score & $\mathbf{2 5}$ slots & Total score & $\mathbf{2 0}$ slots \\
\hline
\end{tabular}

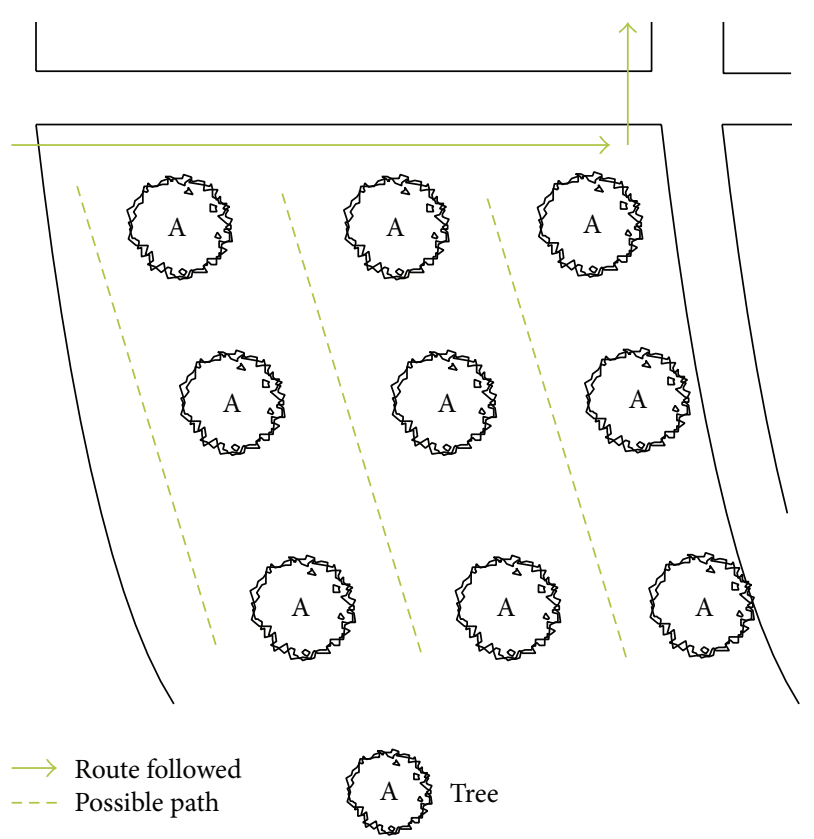

Figure 7: Diagram of the open area.

Next, users had to follow the instructions presented by each application to get to the destination by themselves. Researchers only intervened in case of danger (i.e., crossing without looking) or mistake. In case of error, researchers waited for a short time and, only if users did not recover on their own, they were relocated to the place where they went wrong.

Finally, once the users reported that they had finished, researchers asked them to point to the destination and then escorted them back to the education center. This information (reaching the destination or not and its identification) was also recorded to be included in the subsequent analysis. After finishing all the sessions, one of the educators directed a focus group discussion about their preferred application.

4.2. Data Collection and Analysis. From the videos and registries, 5 measurements were collected for later analysis:

(i) Walking Time. The time taken to walk from the education center to the destination. This factor provides a first estimation of each user's performance, but it has to be considered carefully, since it depends on factors other than users' navigational capabilities, given that pace, waiting time at crossings, and so forth vary according to the situation.

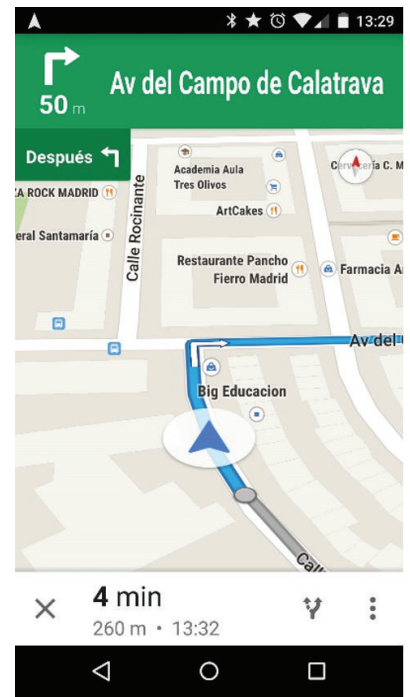

FIGURE 8: Screenshot of Google Maps application at the beginning of the route.

(ii) Reaching the Destination. Whether or not the user reached the final destination. The main goal of any navigational assistant is taking the user to the destination. Therefore, this is the most important factor in the evaluation. Additionally, we considered that reaching the destination also involved identifying it correctly. Therefore, users who did not identify the news-stand or the candy store were counted as not having reached the destination.

(iii) End of Task Recognition. Whether or not the user understood that the destination had been reached.

(iv) Relocations. Taking the wrong direction (and not recovering from the error by themselves). In this case, users were relocated to their last correct position.

(v) Mistakes. Errors made when taking decisions. That is, taking the wrong direction at decision points or not being aware of these points and passing by.

Table 4 summarizes the measured factors for both routes and applications.

As we have said above, the time factor provides a first estimation of users' performance. In this case, Google Maps offered better results than AssisT-OUT. Moreover, this result is supported statistically ( $p=0.011$; Independent Samples Mann-Whitney $U$ Test). Therefore, we can affirm that technology influenced the time factor. However, this time is also 
TABLE 4: Measured factors during the evaluation.

\begin{tabular}{lccc}
\hline & Google Maps & AssisT-Out & $p$ value \\
\hline Walking time $(\mathrm{s})$ & $329.70(79.6)$ & $410.25(91.47)$ & $\mathbf{0 . 0 1 1}$ \\
Reaching the destination $(\mathrm{y} / \mathrm{n})$ & $9 / 9$ & $17 / 1$ & $\mathbf{0 . 0 2 2}$ \\
End of task recognition $(\mathrm{y} / \mathrm{n})$ & $18 / 0$ & $18 / 0$ & 1.000 \\
Relocations & $0.39(1.04)$ & $0.50(0.79)$ & 0.339 \\
Mistakes & $1.17(2.28)$ & $0.56(0.98)$ & 0.767 \\
\hline
\end{tabular}

affected by users' pace, time spent waiting to cross streets, and so forth.

The next measure is the number of users who arrive at the destination. This is the most important factor since the goal of any navigation assistance is to make the user reach the destination. In this case, AssisT-OUT yielded far better results. Seventeen out of the 18 participants reached the destination and identified it correctly. In the case of Google Maps, only 9 out of the 18 did so. This result is supported statistically ( $p=0.022$; Independent Samples Mann-Whitney $U$ Test). Moreover, even if relocations are included in the analysis, AssisT-OUT continues to yield better results.

Regarding the number of errors, AssisT-OUT presented lower values, but no statistical evidence supported this result. Hence, we cannot clearly support one application or the other. However, we can state that AssisT-OUT is, at least, as good as the standard application regarding this factor, which is a positive result. The same occurs in the case of relocations: no statistical evidence was found to support the difference.

Finally, from the discussion group, it emerged that 9 out of the 18 users preferred AssisT-OUT, 4 preferred Google Maps, 4 preferred both equally, and only one user did not have any specific preference. This is a positive result as well, since users chose the adapted proposal over the standard one.

4.3. Discussion and Future Work. As presented in the related work section, previous studies such as that of Liu et al. [12] compared different mechanisms to guide people with cognitive disabilities. Moreover, Goodman et al. [21] and RassmusGröhn and Magnusson [22] conducted similar studies on elderly people with memory problems. Although their focus groups were different, they presented equivalent findings: the best way to orient people with special needs is landmarkbased directions.

These ideas motivated us to design, develop, and evaluate a novel system, based on these works, but providing full functionality. That is, previous experiments were based on Wizard-of-Oz approaches that required external control during the trials or the preparation of routes, pictures, and their processing by researchers or educators. In our case, this process is carried out automatically since the route calculation and street-level photographs are requested from external services and processed at the server in execution time. This way, not only was our approach closer to a realworld deployment, but it also reduced carers' workload.

From the evaluation, we can affirm that our wayfinding proposal, based on atomic instructions and street-level images at decision points, worked better than the commercial application. The most valuable result was the higher number of users who arrived at the destination and identified it correctly. This factor is crucial in navigation and is the main objective of these applications: to enable users to reach their destinations. Related to this, another important issue we found was that some users, despite not finishing the route, reported they had reached the destination (such destinations were not valid). We consider that reaching and recognizing the destination are both equally significant. Therefore, these false identifications are also critical in wayfinding solutions and, what is more, dangerous, since users may feel confused or lost.

Another important metric in evaluating navigation assistants is the time taken to complete the route. In this case, users required significantly more time when using AssistOUT. We consider that this increment in AssisT-OUT times is motivated by the fact that it is a systematic application, which requires users' attention and interaction during the route, while Google Maps offers a more dynamic experience and users only have to interact with it at setup.

At this point we would like to recall the fact that, in the case of Google Maps, the application was given to users preconfigured. That is, a researcher selected the destination and asked the application for the route. In contrast, in the case of AssisT-OUT, users selected the destination by themselves. Therefore, they performed the whole process instead of only the navigation part. According to their educators, many of the users would not have been able to configure Google Maps application by themselves, which would have made the navigation impossible. Thus, not only did AssisT-OUT make more users reach the destination, but it also enabled assistance by means of the adapted interface.

Although the system worked satisfactorily in a real and controlled setting, for intensive use it needs to be improved in terms of route recalculation. Due to GPS accuracy errors, lack of attention, or confusion between left and right, users may deviate from the proposed route and be unable to return to the correct place on their own. Therefore, the route should be recalculated. This feature should be implemented in future versions of the system but in a sensible way. Many commercial systems recalculate the route and propose a new path. In our case, as we want to assist and train users at the same time, it would be preferable to guide them back to the location where they took the wrong direction and continue with the precalculated route.

In the same sense, the public transport system should be included in route calculation. This way, more users could make use of the application in their daily lives. In small cities or walks around the neighborhood, this is not necessary, but it would be useful in bigger cities where users may have to cover several kilometers to get to their workplace or education center.

\section{Conclusions}

In this paper, we have presented a novel wayfinding system adapted for people with cognitive disabilities. The path planning and presentation have been specifically developed to meet their needs. Therefore, instead of showing complex instructions on maps, we decided to break down the route 
into atomic instructions and make use of images at the decision points. This mechanism, supported by location awareness alarms, constitutes a different, more adapted approach in comparison to those offered by standard commercial applications.

From the evaluation, we can conclude that an adapted system such as that we have described here surpasses the performance of a standard navigation tool in terms of the number of users who reached and identified their destination correctly.

Outdoor guidance is a promising research issue for people with cognitive disabilities. Most of these users are not allowed to go alone to new or unfamiliar places. And, as can be seen in the results of the evaluation, the available standard tools do not meet their requirements. Only half of standard navigation tool users reached their destination compared to almost all participants using the adapted version. Only the time to finish the route increased slightly with the adapted tool, which was offset by a higher success ratio.

A dependable, scalable tool that is easy to use and understand is indispensable for providing assurance to people with cognitive disabilities and their relatives in their autonomous movements. We consider that the proposed paradigm, with atomic tasks, simple instructions, and continuous real photos provides a promising approach to accommodate the requirements of these users. The evaluation conducted with users with diverse grades of disability confirms this idea, establishing a focus of research for specifically adapted applications for people with cognitive disabilities affecting their mobility.

\section{Conflict of Interests}

The authors declare that there is no conflict of interests regarding the publication of this paper.

\section{Acknowledgments}

This work has been partially funded by Projects "e-Training y e-Coaching para la Integración Socio-Laboral" (TIN201344586--R) and "eMadrid-CM: Investigación y Desarrollo de Tecnologías Educativas en la Comunidad de Madrid" (S2013/ICE-2715).

\section{References}

[1] E. Foulke, "Perception, cognition and the mobility of blind pedestrians," in Spatial Abilities: Development and Physiological Foundations, pp. 55-76, Academic Press, New York, NY, USA, 1982.

[2] K. Lynch, The Image of the City, vol. 11, MIT Press, 1960.

[3] D. R. Montello and C. Sas, "Human factors of wayfinding in navigation," in International Encyclopedia of Ergonomics and Human Factors, pp. 2003-2008, CRC Press, Taylor \& Francis, 2006.

[4] J. M. Loomis, R. L. Klatzky, R. G. Golledge, and J. W. Philbeck, "Human navigation by path integration," in Wayfinding Behavior: Cognitive Mapping and Other Spatial Processes, pp. 125-151, Johns Hopkins University Press, Baltimore, Md, USA, 1999.
[5] H. Mengue-Topio, Y. Courbois, E. K. Farran, and P. Sockeel, "Route learning and shortcut performance in adults with intellectual disability: a study with virtual environments," Research in Developmental Disabilities, vol. 32, no. 1, pp. 345-352, 2011.

[6] S. E. Stock, D. K. Davies, M. L. Wehmeyer, and Y. Lachapelle, "Emerging new practices in technology to support independent community access for people with intellectual and cognitive disabilities," NeuroRehabilitation, vol. 28, no. 3, pp. 261-269, 2011.

[7] R. Hervás, J. Bravo, and J. Fontecha, "An assistive navigation system based on augmented reality and context awareness for people with mild cognitive impairments," IEEE Journal of Biomedical and Health Informatics, vol. 18, no. 1, pp. 368-374, 2014.

[8] H. Holone, G. Misund, and H. Holmstedt, "Users are doing it for themselves: pedestrian navigation with user generated content," in Proceedings of the International Conference on Next Generation Mobile Applications, Services and Technologies (NGMAST '07), pp. 92-99, September 2007.

[9] M. Rispoli, W. Machalicek, and R. Lang, "Assistive technology for people with acquired brain injury," in Assistive Technologies for People with Diverse Abilities, Autism and Child Psychopathology Series, pp. 21-52, Springer, New York, NY, USA, 2014.

[10] A. K. Beeharee and A. Steed, "A natural wayfinding exploiting photos in pedestrian navigation systems," in Proceedings of the 8th International Conference on Human-Computer Interaction with Mobile Devices and Services (MobileHCI '06), pp. 81-88, Espoo, Finland, September 2006.

[11] S. Fickas, M. Sohlberg, and P.-F. Hung, "Route-following assistance for travelers with cognitive impairments: a comparison of four prompt modes," International Journal of Human Computer Studies, vol. 66, no. 12, pp. 876-888, 2008.

[12] A. L. Liu, H. Hile, G. Borriello et al., "Customizing directions in an automated wayfinding system for individuals with cognitive impairment," in Proceedings of the 11th International ACM SIGACCESS Conference on Computers and Accessibility (ASSETS '09), pp. 27-34, Pittsburgh, Pa, USA, October 2009.

[13] R. Lemoncello, M. Moore Sohlberg, and S. Fickas, "How best to orient travellers with acquired brain injury: a comparison of three directional prompts," Brain Injury, vol. 24, no. 3, pp. 541549, 2010.

[14] H. Kaminoyama, T. Matsuo, F. Hattori, K. Susami, N. Kuwahara $\mathrm{y}$, and S. Abe, "Walk navigation system using photographs for people with dementia," in Human Interface and the Management of Information. Interacting in Information Environments, vol. 4558 of Lecture Notes in Computer Science, pp. 1039-1049, Springer, Berlin, Germany, 2007.

[15] A. G. García de Marina, R. M. Carro, and P. Haya, "Where should I go?: guiding users with cognitive limitations through mobile devices outdoors," in Proceedings of the 13th International Conference on Interacción Persona-Ordenador, Elche, Spain, October 2012.

[16] S. Fickas, R. Lemoncello, and M. Sohlberg, "Where am I: how travelers with a cognitive impairment ask for and use help," in Proceedings of the 1st International Workshop on User Modeling and Adaptation for Daily Routines: Providing Assistance to People with Special and Specific Needs, pp. 5-18, Hawaii, Hawaii, USA, June 2010.

[17] N. Fallah, I. Apostolopoulos, K. Bekris, and E. Folmer, "Indoor human navigation systems: a survey," Interacting with Computers, vol. 25, no. 1, pp. 21-33, 2013. 
[18] M. G. Friedman and D. N. Bryen, "Web accessibility design recommendations for people with cognitive disabilities," Technology and Disability, vol. 19, no. 4, pp. 205-212, 2007.

[19] J. Gomez and T. Ojala, "A mobile navigation system based on visual cues for pedestrians with cognitive disabilities," in Assistive Technologies for Physical and Cognitive Disabilities, pp. 173190, 2014.

[20] K.-F. Richter and M. Duckham, "Simplest instructions: finding easy-to-describe routes for navigation," in Geographic Information Science, pp. 274-289, Springer, 2008.

[21] J. Goodman, S. A. Brewster, and P. Gray, "How can we best use landmarks to support older people in navigation?" Behaviour \& Information Technology, vol. 24, no. 1, pp. 3-20, 2005.

[22] K. Rassmus-Gröhn and C. Magnusson, "Finding the way homesupporting wayfinding for older users with memory problems," in Proceedings of the 8th Nordic Conference on HumanComputer Interaction: Fun, Fast, Foundational (NordiCHI '14), pp. 247-255, October 2014. 

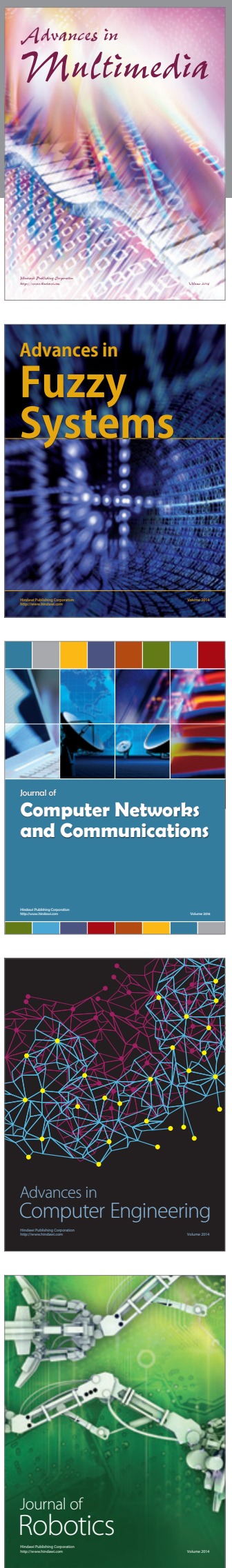

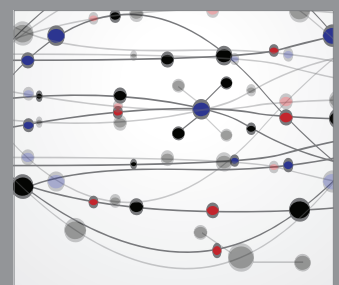

The Scientific World Journal
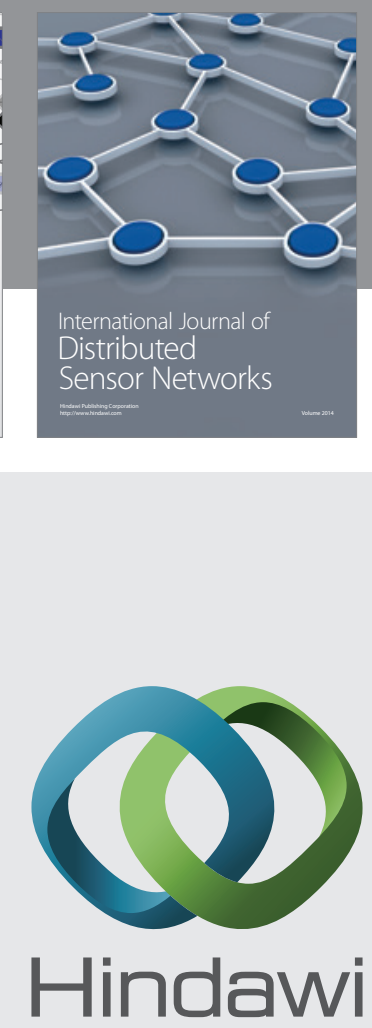

Submit your manuscripts at

http://www.hindawi.com
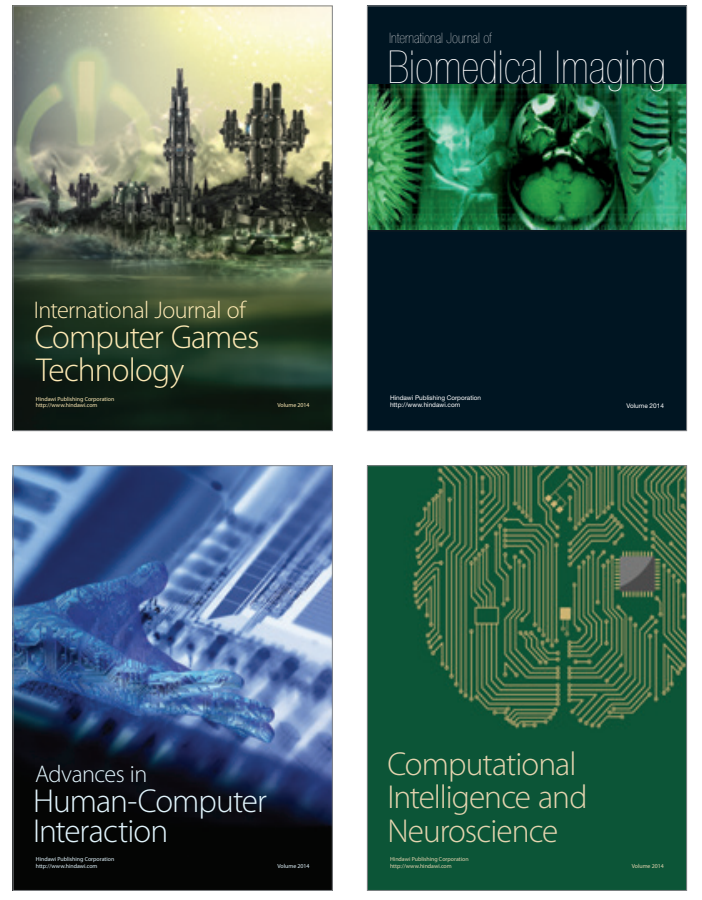
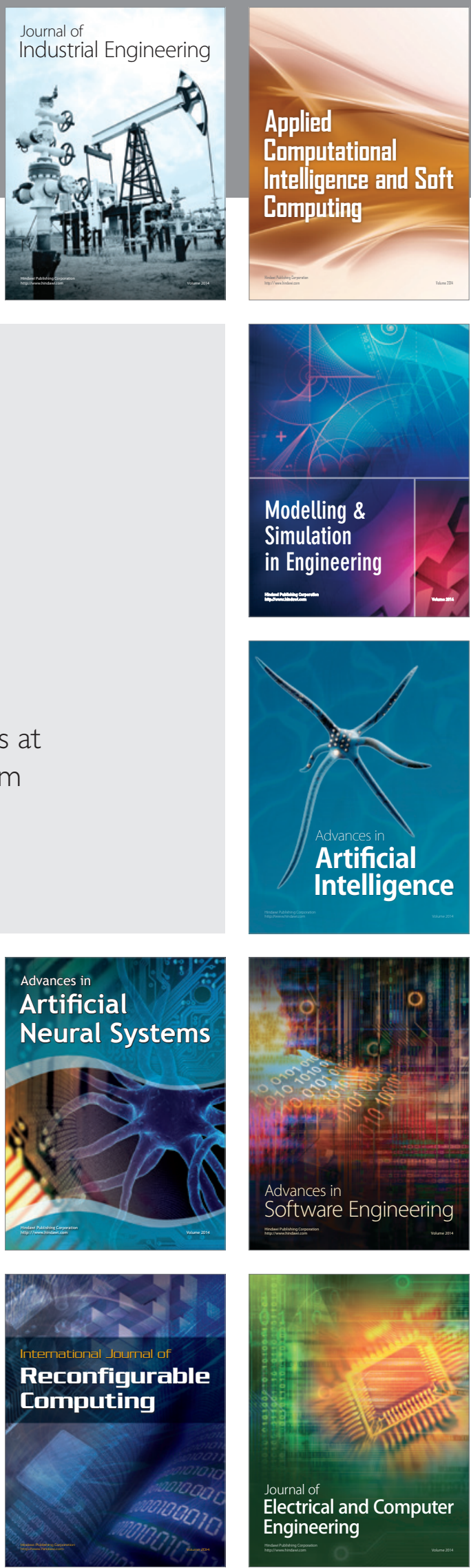